\title{
THE COMPLEX MONGE-AMPÈRE EQUATION WITH A GRADIENT TERM
}

\author{
VALENTINO TOSATTI AND BEN WEINKOVE \\ Dedicated to Professor D.H. Phong on the occasion of his 65th birthday
}

\begin{abstract}
We consider the complex Monge-Ampère equation with an additional linear gradient term inside the determinant. We prove existence and uniqueness of solutions to this equation on compact Hermitian manifolds.
\end{abstract}

\section{INTRODUCTION}

Let $M$ be a compact complex manifold of complex dimension $n$. When $M$ admits a Kähler metric $g=\left(g_{i \bar{j}}\right)$, Yau [35] proved the now classic result that the complex Monge-Ampère equation

$$
\operatorname{det}\left(g_{i \bar{j}}+u_{i \bar{j}}\right)=e^{F} \operatorname{det}\left(g_{i \bar{j}}\right), \quad\left(g_{i \bar{j}}+u_{i \bar{j}}\right)>0,
$$

admits a unique solution $u$ with $\sup _{M} u=0$, as long as $F$ is normalized so that $\left(e^{F}-1\right)$ has zero integral. Equivalently, one can prescribe the volume form of a Kähler metric within a given Kähler class.

Yau's result has been extended and built on in various ways. Modulo adding a constant to $F$, the equation (1.1) can be solved for $g$ Hermitian (by work of Cherrier [6] and the authors [30, see also [16, 29]) and for $g$ almost Hermitian (Chu-Tosatti-Weinkove [7]). Fu-Wang-Wu [1, 12] considered the Monge-Ampère equation obtained by taking the determinant of the $(n-$ $1, n-1)$ form

$$
\omega^{n-1}+\sqrt{-1} \partial \bar{\partial} u \wedge \omega^{n-2}
$$

This is the natural equation on compact manifolds associated to HarveyLawson's notion of $(n-1)$-plurisubharmonicity [18], and was solved for $\omega$ Hermitian by the authors [31, 33. Building on this work, SzékelyhidiTosatti-Weinkove [27] proved existence of solutions for Monge-Ampère equation associated to

$$
\omega^{n-1}+\sqrt{-1} \partial \bar{\partial} u \wedge \omega^{n-2}+L(x, \nabla u),
$$

for the specific first order term

$$
L(x, \nabla u)=\operatorname{Re}\left(\sqrt{-1} \partial u \wedge \bar{\partial} \omega^{n-2}\right)
$$

Partially supported by NSF grants DMS-1610278 (V.T.) and DMS-1709544 (B.W.). Part of this work was done while the first-named author was visiting the Center for Mathematical Sciences and Applications at Harvard University, which he thanks for the hospitality. 
introduced by Popovici [25] and independently in [33. This yielded a solution of the Gauduchon conjecture [15] on the existence of Gauduchon metrics with prescribed volume form. The proof in [27] makes careful use of the specific form of this first order term term $L(x, \nabla u)$. See also [17, 10, 26, 38] for related follow-up work.

Other nonlinear equations involving gradient terms arise naturally by motivations from mathematical physics, including the Fu-Yau equation 13 and its extensions by Phong-Picard-Zhang [21, 22, 23]. In particular, the paper [21] considers the complex Hessian equations

$$
(\chi(z, u)+\sqrt{-1} \partial \bar{\partial} u)^{k} \wedge \omega^{n-k}=\psi(z, u, \nabla u) \omega^{n}
$$

where gradient terms appear on the right hand side.

In light of these results, it is natural to consider fully nonlinear equations in terms of the metric

$$
\tilde{\omega}=\omega+\sqrt{-1} \partial \bar{\partial} u+L(x, \nabla u)
$$

for $L$ a linear term involving the gradient of $u$. Indeed, this study was initiated recently by R. Yuan [36. However the family of equations he deals with includes the Monge-Ampère equation $\tilde{\omega}^{n}=e^{F} \omega^{n}$ only in the case of complex dimension $n=2$ [36, Corollary 1.5]. The current paper settles the case $n>2$ left open by Yuan.

More precisely, let $(M, g)$ be a compact Hermitian manifold of complex dimension $n$. By analogy to (1.2), we consider the term

$$
L(x, \nabla u)=\sqrt{-1} a \wedge \bar{\partial} u-\sqrt{-1} \bar{a} \wedge \partial u
$$

where $a$ is a smooth $(1,0)$-form. Indeed, this is the most general term of the form $\alpha \wedge \partial u+\beta \wedge \bar{\partial} u$ for 1 -forms $\alpha$ and $\beta$, which is also real and of type $(1,1)$. In local coordinates, we may write $L(x, \nabla u)=\sqrt{-1}\left(a_{i} u_{\bar{j}}+a_{\bar{j}} u_{i}\right) d z^{i} \wedge d \bar{z}^{j}$, where $a=a_{i} d z^{i}$ and $a_{\bar{i}}=\overline{a_{i}}$.

We prove the following:

Theorem 1.1. Given $F \in C^{\infty}(M)$ and a smooth $(1,0)$ form a on $M$, there exists a unique pair $(u, b)$ with $u \in C^{\infty}(M)$ and $b \in \mathbb{R}$ satisfying the equation

$$
\begin{aligned}
& \operatorname{det}\left(g_{i \bar{j}}+a_{i} u_{\bar{j}}+a_{\bar{j}} u_{i}+u_{i \bar{j}}\right)=e^{F+b} \operatorname{det}\left(g_{i \bar{j}}\right), \\
& \text { with }\left(\tilde{g}_{i \bar{j}}\right):=\left(g_{i \bar{j}}+a_{i} u_{\bar{j}}+a_{\bar{j}} u_{i}+u_{i \bar{j}}\right)>0, \quad \text { and } \sup _{M} u=0 .
\end{aligned}
$$

The case $n=2$ is due to Yuan [36. We also remark that Zhang 37] proved a uniform gradient estimate for a class of equations which includes (1.3).

We can rewrite (1.3) in coordinate-free notation by letting

$$
\tilde{\omega}:=\omega+\sqrt{-1} a \wedge \bar{\partial} u-\sqrt{-1} \bar{a} \wedge \partial u+\sqrt{-1} \partial \bar{\partial} u>0,
$$

be the new Hermitian metric whose volume form equals

$$
\tilde{\omega}^{n}=e^{F+b} \omega^{n} .
$$


Remark 1.2. As an aside, note that if we choose $a$ to be a holomorphic 1 -form, then we can write

$$
\tilde{\omega}=\omega+\partial \bar{\gamma}+\bar{\partial} \gamma
$$

where $\gamma$ is the $(1,0)$ form given by

$$
\gamma=-\sqrt{-1}\left(u a+\frac{\partial u}{2}\right)
$$

In this case, if we also have that $\partial \bar{\partial} \omega=0$ (which when $n=2$ is the Gauduchon condition [14]), then $\omega$ defines a cohomology class in Aeppli cohomology, and (1.4) shows that the metric $\tilde{\omega}$ also satisfies $\partial \bar{\partial} \tilde{\omega}=0$ and lies in the same Aeppli cohomology class.

The outline of our proof is as follows. We begin by proving a priori estimates for solutions of (1.3). In Section 2, we establish a uniform $L^{\infty}$ bound for $u$, with an approach that uses the Aleksandrov-Bakelman-Pucci estimate. In Section 3 we give an estimate on the second derivatives $\sqrt{-1} \partial \bar{\partial} u$ of $u$ in terms of the first derivatives, using a maximum principle argument involving the largest eigenvalue $\lambda_{1}$ of the metric $\tilde{g}$. The particular quantity we use for the maximum principle is

$$
Q=\log \lambda_{1}+\frac{|\partial u|_{g}^{2}}{\sup _{M}|\partial u|_{g}^{2}+1}+e^{-A u}
$$

for a large constant $A$. This differs (and in many cases is simpler) than the quantities used in the literature mentioned above. To overcome the fact that the eigenvalue $\lambda_{1}$ is not differentiable in general, we choose to use a viscosity argument (adapted from [5], and hinted to in [26]), which to our knowledge is new in this Hermitian setting. Finally, in Section 4, we complete the proof of Theorem 1.1 we apply a standard blow-up argument to obtain the first order estimate and then standard theory gives the higher order estimates. Given the $C^{\infty}$ a priori estimates, the existence follows from a fairly standard continuity argument and uniqueness is a consequence of the maximum principle.

Instead of using a blow-up argument, the gradient estimate can be obtained directly by a maximum principle argument, as shown in an earlier work of Zhang [37, Remark 2] (see also the related works [4, 10, 36]). We thank the referee for pointing out the reference [37, of which we were not aware when we completed the first version of this article.

Acknowledgments. Both authors owe many thanks to Professor Phong, to whom this article is dedicated. His mathematical wisdom and insights are an inspiration to us. Happy birthday Phong! 


\section{ZERo ORDER ESTIMATE}

Let $u, F \in C^{\infty}(M)$ and $a \in \Lambda^{1,0} M$ satisfy

$$
\begin{gathered}
\operatorname{det}\left(g_{i \bar{j}}+a_{i} u_{\bar{j}}+a_{\bar{j}} u_{i}+u_{i \bar{j}}\right)=e^{F} \operatorname{det}\left(g_{i \bar{j}}\right) \\
\left(\tilde{g}_{i \bar{j}}\right):=\left(g_{i \bar{j}}+a_{i} u_{\bar{j}}+a_{\bar{j}} u_{i}+u_{i \bar{j}}\right)>0,
\end{gathered}
$$

with $\sup _{M} u=0$. We will write $\tilde{\omega}$ for the $(1,1)$ form associated to the metric $\tilde{g}_{i \bar{j}}$.

We prove a uniform estimate for $u$.

Theorem 2.1. There is a constant $C$ that depends only on $\sup _{M}|F|, \sup _{M}|a|_{g}$, and on the geometry of $(M, g)$ such that

$$
\sup _{M}|u| \leqslant C
$$

Proof. We employ the Aleksandrov-Bakelman-Pucci estimate, whose usage for the complex Monge-Ampère equation originated in work of Cheng-Yau (see [1]), and was more recently revisited by Błocki [2, 3] and Székelyhidi [26. We follow [7, 26, 32].

First, we observe that

$$
\int_{M}(-u) \omega^{n} \leqslant C
$$

for a uniform constant $C$. Indeed, let

$$
H(u)=\Delta_{g} u+\operatorname{tr}_{\omega}(\sqrt{-1} a \wedge \bar{\partial} u-\sqrt{-1} \bar{a} \wedge \partial u)=\operatorname{tr}_{g} \tilde{g}-n \geqslant-n,
$$

where $\Delta_{g} u=\operatorname{tr}_{\omega} \sqrt{-1} \partial \bar{\partial} u=\frac{n \sqrt{-1} \partial \bar{\partial} u \wedge \omega^{n-1}}{\omega^{n}}$ is the complex Laplacian of $g$. Since the kernel of $H$ consists of just constants, a classical argument of Gauduchon [14] (cf. [7, Theorem 2.2]) shows that there is a smooth function $v$ such that

$$
\int_{M} H(\psi) e^{v} \omega^{n}=0
$$

for all smooth functions $\psi$. We then define a new Hermitian metric $\hat{\omega}=$ $e^{v /(n-1)} \omega$. Its operator $\hat{H}$, defined in the same way

$$
\hat{H}(\psi)=\Delta_{\hat{g}} \psi+\operatorname{tr}_{\hat{\omega}}(\sqrt{-1} a \wedge \bar{\partial} \psi-\sqrt{-1} \bar{a} \wedge \partial \psi),
$$

satisfies

$$
\hat{H}(u)=e^{-v /(n-1)} H(u) \geqslant-C,
$$

and now we have

$$
\int_{M} \hat{H}(\psi) \hat{\omega}^{n}=0
$$

for all $\psi$. We may then use the Green's function for $\hat{H}$ (with respect to the metric $\hat{\omega}$ ), to deduce the uniform $L^{1}$ bound for $u$ in (2.3) by the exact same argument as in [33, Proof of Theorem 2.1]. Briefly, standard theory gives 
us a Green's function $G(x, y)$, normalized to have zero integral, which has a uniform lower bound and such that

$$
\psi(x)=\frac{1}{\int_{M} \hat{\omega}^{n}} \int_{M} \psi \hat{\omega}^{n}-\int_{M} \hat{H}(\psi)(y) G(x, y) \hat{\omega}^{n}(y),
$$

holds for all $\psi$ and all $x \in M$. Thanks to (2.7) we can add a uniform constant to $G$ to make it nonnegative, while preserving the same Green's formula, and we then apply this to $u$ with $x$ a point where $u(x)=0$, so that from (2.6) and the lower bound for $G$ we easily deduce (2.3).

Next, we promote the $L^{1}$ bound (2.3) to the $L^{\infty}$ bound (2.2) using ABP, as in [7, Proposition 3.1] and [26, 32]. Let $x_{0} \in M$ be a point where $u$ achieves its infimum $I=\inf _{M} u$, and fix a coordinate unit ball $B$ centered at $x_{0}$. In this ball, let $v=u+\varepsilon|x|^{2}$, where $\varepsilon>0$ will be a uniform constant to be chosen later. We have $\inf _{\partial B} v \geqslant v(0)+\varepsilon$, so [26, Proposition 10] gives us that

$$
\varepsilon^{2 n} \leqslant C \int_{P} \operatorname{det}\left(D^{2} v\right)
$$

for a universal constant $C$, where

$$
P=\{x \in B|| D v(x) \mid<\varepsilon / 2 \text {, and } v(y) \geqslant v(x)+D v(x) \cdot(y-x) \forall y \in B\} .
$$

Given now any $x \in P$, we have $D^{2} v(x) \geqslant 0$ and $|D u(x)| \leqslant 5 \varepsilon / 2$ so at $x$

$$
\sqrt{-1} a \wedge \bar{\partial} u-\sqrt{-1} \bar{a} \wedge \partial u+\sqrt{-1} \partial \bar{\partial} u \geqslant-C \varepsilon \omega,
$$

for a uniform constant $C$, therefore if we choose $\varepsilon$ sufficiently small (but uniformly bounded away from zero), we get

$$
\tilde{\omega}(x) \geqslant \frac{1}{2} \omega(x)
$$

and from the Monge-Ampère equation (2.1) we deduce

$$
\tilde{\omega}(x) \leqslant C \omega(x),
$$

from which

$$
\sqrt{-1} \partial \bar{\partial} u(x) \leqslant C \omega(x)
$$

and so $0 \leqslant \sqrt{-1} \partial \bar{\partial} v(x) \leqslant C \omega(x)$. But a simple linear algebra inequality (using that $\left(D^{2} v(x)\right) \geqslant 0$ ) gives

$$
\operatorname{det}\left(D^{2} v(x)\right) \leqslant C \operatorname{det}\left(v_{i \bar{j}}\right)^{2}(x) \leqslant C,
$$

which together with (2.8) gives

$$
|P| \geqslant C^{-1},
$$

where $|P|$ denotes the Lebesgue measure. For all $x \in P$ we have

$$
v(x) \leqslant v(0)+\frac{\varepsilon}{2}=I+\frac{\varepsilon}{2},
$$

and we may assume that $I+\frac{\varepsilon}{2}<0$, so

$$
C^{-1} \leqslant|P| \leqslant \frac{\int_{P}(-v)}{\left|I+\frac{\varepsilon}{2}\right|} \leqslant \frac{C}{\left|I+\frac{\varepsilon}{2}\right|},
$$


using the $L^{1}$ bound (2.3) , which proves (2.2).

\section{Second order estimate}

In this section we prove a bound on $\sqrt{-1} \partial \bar{\partial} u$ in terms of a bound on the square of the first derivative of $u$. This estimate takes the same form as the Hou-Ma-Wu estimate [19] for the complex Hessian equations (see also the later works [7, 26, 27, 31, 33]) although here the quantity to which we apply the maximum principle is slightly simpler.

Theorem 3.1. Let $u, F \in C^{\infty}(M)$ and $a \in \Lambda^{1,0} M$ satisfy (2.1), with $\sup _{M} u=0$. Then there is a constant $C$ that depends only on $\sup _{M}|u|$, $\|a\|_{C^{2}(M)},\|F\|_{C^{2}(M)}$ and on the geometry of $(M, g)$ such that

$$
\sup _{M}|\sqrt{-1} \partial \bar{\partial} u|_{g} \leqslant C\left(1+\sup _{M}|\partial u|_{g}^{2}\right) .
$$

Proof. Define the linearized operator $L$ by

$$
L v=\tilde{g}^{i \bar{j}}\left(v_{i \bar{j}}+a_{i} v_{\bar{j}}+a_{\bar{j}} v_{i}\right)=\tilde{g}^{i \bar{j}} v_{i \bar{j}}+2 \operatorname{Re}\left(\tilde{g}^{i \bar{j}} a_{\bar{j}} v_{i}\right) .
$$

Observe that

$$
L u=\tilde{g}^{i \bar{j}}\left(\tilde{g}_{i \bar{j}}-g_{i \bar{j}}\right)=n-\operatorname{tr}_{\tilde{g}} g .
$$

Let $\lambda_{1} \geqslant \lambda_{2} \geqslant \cdots \geqslant \lambda_{n}>0$ be the eigenvalues of $\tilde{g}_{i \bar{j}}$ with respect to $g$. We consider the quantity

$$
Q=\log \lambda_{1}+\varphi\left(|\partial u|_{g}^{2}\right)+\psi(u),
$$

where we define

$$
\varphi(s)=\frac{s}{K}, s \geqslant 0, \quad \text { and } \quad \psi(t)=e^{-A t}, t \leqslant 0,
$$

with

$$
K=\sup _{M}|\partial u|_{g}^{2}+1,
$$

and $A>0$ to be determined. Note that we have

$$
-\psi^{\prime} \geqslant A>0, \quad \psi^{\prime \prime}=-A \psi^{\prime} .
$$

We assume that $Q$ achieves its maximum at $x_{0} \in M$. It suffices to show that at $x_{0}$, we have $\lambda_{1} \leqslant C K$ for a uniform $C$. Hence in what follows we may assume without loss of generality that $\lambda_{1}$ is large compared to $K$. We will calculate at the point $x_{0}$ using coordinates for which $g$ is the identity and $\tilde{g}$ is diagonal with entries $\tilde{g}_{i \bar{i}}=\lambda_{i}$ for $i=1, \ldots, n$.

Since $\lambda_{1}$ may not be smooth at $x_{0}$, we define a smooth function $f$ on $M$ by (cf. [5, Proof of Theorem 6])

$$
Q\left(x_{0}\right)=\log f+\varphi\left(|\partial u|_{g}^{2}\right)+\psi(u)
$$

where the right hand side of (3.3) is evaluated at a general point of $M$. Observe that $f$ satisfies

$$
f \geqslant \lambda_{1} \quad \text { on } M, \quad f=\lambda_{1} \quad \text { at } x_{0} .
$$


We have the following lemma, which is a complex version of [5, Lemma 5]. Here and in the sequel, we use $\nabla_{i}$ or simply lower indices (after commas, when needed to avoid confusion) to denote covariant derivatives with respect to the Chern connection of $g$.

Lemma 3.2. Let $\mu$ denote the multiplicity of the largest eigenvalue of $\tilde{g}$ at $x_{0}$, so that $\lambda_{1}=\cdots=\lambda_{\mu}>\lambda_{\mu+1} \geqslant \cdots \geqslant \lambda_{n}$. Then at $x_{0}$, for each $i$ with $1 \leqslant i \leqslant n$,

$$
\tilde{g}_{k \bar{\ell}, i}=f_{i} g_{k \bar{\ell}}, \quad \text { for } 1 \leqslant k, \ell \leqslant \mu,
$$

and

$$
f_{i \bar{i}} \geqslant \tilde{g}_{1 \overline{1}, i \bar{i}}+\sum_{q>\mu} \frac{\left|\tilde{g}_{q \overline{1}, i}\right|^{2}+\left|\tilde{g}_{q \overline{1}, \bar{i}}\right|^{2}}{\lambda_{1}-\lambda_{q}} .
$$

Proof. The proof only uses the fact that $f$ is smooth and satisfies (3.4). For a smooth vector field $V=V^{k} \frac{\partial}{\partial z^{k}}$ defined in a neighborhood of $x_{0}$, we consider the function

$$
h=\tilde{g}_{k \bar{\ell}} V^{k} \overline{V^{\ell}}-f g_{k \bar{\ell}} V^{k} \overline{V^{\ell}},
$$

which is nonpositive. For any choice of $V$ with $V^{k}\left(x_{0}\right)=0$ for $k>\mu$ we have $h\left(x_{0}\right)=0$ and hence $h$ has a local maximum at $x_{0}$.

For (3.5), choose $V$ with $V^{k}\left(x_{0}\right)=0$ for $k>\mu$ and

$$
\nabla_{i} V^{k}\left(x_{0}\right)=0=\nabla_{\bar{i}} V^{k}\left(x_{0}\right), \quad \text { for } k \leqslant \mu .
$$

Then at $x_{0}$,

$$
0=h_{i}=\tilde{g}_{k \bar{\ell}, i} V^{k} \overline{V^{\ell}}-f_{i} g_{k \bar{\ell}} V^{k} \overline{V^{\ell}},
$$

and (3.5) follows since we can choose $V^{k}\left(x_{0}\right)$ for $k \leqslant \mu$ to be whatever we like.

For (3.6) we choose $V$ with $V\left(x_{0}\right)=\frac{\partial}{\partial z^{1}}$ and

$$
\nabla_{i} V^{q}\left(x_{0}\right)= \begin{cases}0, & q \leqslant \mu \\ \frac{\tilde{g}_{1 \bar{q}, i}}{\lambda_{1}-\lambda_{q}}, & q>\mu\end{cases}
$$

and

$$
\nabla_{\bar{i}} V^{q}\left(x_{0}\right)= \begin{cases}0, & q \leqslant \mu \\ \frac{\tilde{g}_{1 \bar{q}, \bar{i}}}{\lambda_{1}-\lambda_{q}}, & q>\mu .\end{cases}
$$

Then at $x_{0}$,

$$
\begin{aligned}
0 \geqslant h_{i \bar{i}}= & \tilde{g}_{1 \overline{1}, i \bar{i}}-f_{i \bar{i}}+\tilde{g}_{k \bar{\ell}, i}\left(\nabla_{\bar{i}} V^{k}\right) \overline{V^{\ell}}+\tilde{g}_{k \bar{\ell}, i} V^{k} \overline{\nabla_{i} V^{\ell}}+\tilde{g}_{k \bar{\ell}, \bar{i}}\left(\nabla_{i} V^{k}\right) \overline{V^{\ell}} \\
& +\tilde{g}_{k \bar{\ell}, \bar{i}} V^{k} \overline{\nabla_{\bar{i}} V^{\ell}}+\tilde{g}_{k \bar{\ell}} \nabla_{i} V^{k} \overline{\nabla_{i} V^{\ell}}+\tilde{g}_{k \bar{\ell}} \nabla_{\bar{i}} V^{k} \overline{\nabla_{\bar{i}} V^{\ell}} \\
& -f g_{k \bar{\ell}} \nabla_{i} V^{k} \overline{\nabla_{i} V^{\ell}}-f g_{k \bar{\ell}} \nabla_{\bar{i}} V^{k} \overline{\nabla_{\bar{i}} V^{\ell}}
\end{aligned}
$$


noting that terms of the type $f_{i} g_{k \bar{\ell}}\left(\nabla_{\bar{i}} V^{k}\right) \overline{V^{\ell}}$ vanish by definition of $V$ and $\tilde{g}_{k \bar{\ell}}\left(\nabla_{\bar{i}} \nabla_{i} V^{k}\right) \overline{V^{\ell}}-f g_{k \bar{\ell}}\left(\nabla_{\bar{i}} \nabla_{i} V^{k}\right) \overline{V^{\ell}}=0=\tilde{g}_{k \bar{\ell}} V^{k} \overline{\nabla_{i} \nabla_{\bar{i}} V^{\ell}}-f g_{k \bar{\ell}} V^{k} \overline{\nabla_{i} \nabla_{\bar{i}} V^{\ell}}$ since $f g_{1 \overline{1}}=\lambda_{1}=\tilde{g}_{1 \overline{1}}$ at $x_{0}$. Continuing from (3.7), using the definition of $V$,

$$
\begin{aligned}
0 \geqslant & \tilde{g}_{1 \overline{1}, i \bar{i}}-f_{i \bar{i}}+2 \sum_{q>\mu} \frac{\left|\tilde{g}_{q \overline{1}, i}\right|^{2}}{\lambda_{1}-\lambda_{q}}+2 \sum_{q>\mu} \frac{\left|\tilde{g}_{q \overline{1}, \bar{i}}\right|^{2}}{\lambda_{1}-\lambda_{q}} \\
& +\sum_{q>\mu} \lambda_{q} \frac{\left|\tilde{g}_{1 \bar{q}, i}\right|^{2}}{\left(\lambda_{1}-\lambda_{q}\right)^{2}}+\sum_{q>\mu} \lambda_{q} \frac{\left|\tilde{g}_{1 \bar{q}, \bar{i}}\right|^{2}}{\left(\lambda_{1}-\lambda_{q}\right)^{2}} \\
& -\lambda_{1} \sum_{q>\mu} \frac{\left|\tilde{g}_{1 \bar{q}, i}\right|^{2}}{\left(\lambda_{1}-\lambda_{q}\right)^{2}}-\lambda_{1} \sum_{q>\mu} \frac{\left|\tilde{g}_{1 \bar{q}, \bar{i}}\right|^{2}}{\left(\lambda_{1}-\lambda_{q}\right)^{2}} \\
= & \tilde{g}_{1 \overline{1}, i \bar{i}}-f_{i \bar{i}}+\sum_{q>\mu} \frac{\left|\tilde{g}_{q \overline{1}, i}\right|^{2}+\left|\tilde{g}_{q \overline{1}, \bar{i}}\right|^{2}}{\lambda_{1}-\lambda_{q}}
\end{aligned}
$$

as required.

Differentiating (2.1) we obtain

$$
\tilde{g}^{i \bar{i}} \tilde{g}_{\bar{i} \bar{i}, k}=\tilde{g}^{i \bar{i}}\left(u_{i \bar{i} k}+a_{i, k} u_{\bar{i}}+a_{i} u_{k \bar{i}}+a_{\bar{i}, k} u_{i}+a_{\bar{i}} u_{i k}\right)=F_{k},
$$

where here and henceforth we are computing at the point $x_{0}$. Differentiating again, and setting $k=1$,

$$
\tilde{g}^{i \bar{i}} \tilde{g}_{i \bar{i}, 1 \overline{1}}-\tilde{g}^{i \bar{i}} \tilde{g}^{j \bar{j}} \tilde{g}_{i \bar{j}, 1} \tilde{g}_{j \bar{i}, \overline{1}}=F_{1 \overline{1}} .
$$

Now apply $\nabla_{i}$ to the defining equation (3.3) of $f$ to obtain

$$
0=\frac{f_{i}}{\lambda_{1}}+\varphi^{\prime}\left(u_{p} u_{\bar{p} i}+u_{p i} u_{\bar{p}}\right)+\psi^{\prime} u_{i} .
$$

Next apply the operator $L$, as defined in (3.1), to the defining equation of $f$ to obtain,

$$
\begin{aligned}
0= & \frac{\tilde{g}^{i \bar{i}} f_{i \bar{i}}}{\lambda_{1}}-\frac{\tilde{g}^{i \bar{i}}\left|f_{i}\right|^{2}}{\lambda_{1}^{2}}+\varphi^{\prime} \sum_{p} \tilde{g}^{i \bar{i}}\left(\left|u_{p \bar{i}}\right|^{2}+\left|u_{p i}\right|^{2}\right) \\
& +\varphi^{\prime} \tilde{g}^{i \bar{i}}\left(u_{p i \bar{i}} u_{\bar{p}}+u_{\bar{p} i \bar{i}} u_{p}\right)+\psi^{\prime \prime} \tilde{g}^{i \bar{i}}\left|u_{i}\right|^{2}+\psi^{\prime}\left(n-\operatorname{tr}_{\tilde{g}} g\right) \\
& +2 \operatorname{Re}\left(\tilde{g}^{i \bar{i}} a_{\bar{i}} \frac{f_{i}}{\lambda_{1}}\right)+2 \varphi^{\prime} \operatorname{Re}\left(\tilde{g}^{i \bar{i}} a_{\bar{i}}\left(u_{p} u_{\bar{p} i}+u_{p i} u_{\bar{p}}\right)\right),
\end{aligned}
$$

where we have made use of (13.2). We wish to compare $\sum_{i} \tilde{g}^{i \bar{i}} f_{i \bar{i}}$ and $\sum_{i} \tilde{g}^{i \bar{i}} \tilde{g}_{i \bar{i}, 1 \overline{1}}$. From Lemma 3.2 ,

$$
f_{i}=\tilde{g}_{11, \bar{i}}, \text { and } f_{i \bar{i}} \geqslant \tilde{g}_{1 \overline{1}, i \bar{i}}+\sum_{q>\mu} \frac{\left|\tilde{g}_{1 \bar{q}, i}\right|^{2}+\left|\tilde{g}_{q \overline{1}, i}\right|^{2}}{\lambda_{1}-\lambda_{q}} .
$$


To compare $\tilde{g}_{11, i \bar{i}}$ and $\tilde{g}_{i \bar{i}, 1 \overline{1}}$ we first compute, using $T_{i j}^{k}$ and $R_{k \bar{\ell} i}{ }^{p}$ to denote the torsion and Chern curvature tensors of $g$ respectively (see for example [33]),

$$
\begin{aligned}
& u_{i \bar{i} \overline{1} \overline{1}}=u_{i \bar{i} \overline{1} 1}+R_{1 \overline{1} i}^{p} u_{p \bar{i}}-R_{1 \overline{1} \bar{i}}^{\bar{q}} u_{i \bar{q}} \\
& =u_{i \overline{1 i} 1}+R_{1 \overline{1} i}{ }^{p} u_{p \bar{i}}-R_{1 \overline{1}{ }^{\bar{q}}} u_{i \bar{q}}+\nabla_{1} \overline{T_{i 1}^{q}} u_{i \bar{q}}+\overline{T_{i 1}^{q}} u_{i \bar{q} 1} \\
& =u_{\overline{1} i 1 \bar{i}}+R_{1 \overline{1} i}{ }^{p} u_{p \bar{i}}-R_{1 \overline{1}{ }_{\bar{i}}}^{\bar{q}} u_{i \bar{q}}+\nabla_{1} \overline{T_{i 1}^{q}} u_{i \bar{q}}+\overline{T_{i 1}^{q}} u_{i \bar{q} 1} \\
& +R_{1 \bar{i}} \overline{\bar{q}}_{\overline{1}} u_{\bar{q} i}-R_{1 \bar{i} i}^{p} u_{\overline{1} p} \\
& =u_{1 \overline{1} i \bar{i}}+R_{1 \overline{1} i}{ }^{p} u_{p \bar{i}}-R_{1 \overline{1} \bar{i}}^{\bar{q}} u_{i \bar{q}}+\nabla_{1} \overline{T_{i 1}^{q}} u_{i \bar{q}}+\overline{T_{i 1}^{q}} u_{i \bar{q} 1} \\
& +R_{1 \bar{i} \overline{1}}{ }^{\bar{q}} u_{\bar{q} i}-R_{1 \bar{i} i}^{p} u_{\overline{1} p}+\nabla_{\bar{i}} T_{i 1}^{q} u_{\overline{1} q}+T_{i 1}^{q} u_{\overline{1} q \bar{i}} \text {, }
\end{aligned}
$$

where for the second inequality and fourth inequalities, we used the formulae

$$
u_{j \overline{\ell k}}-u_{j \overline{k \ell}}=\overline{T_{\ell k}^{q}} u_{j \bar{q}}, \quad u_{\bar{j} \ell k}-u_{\bar{j} k \ell}=T_{\ell k}^{q} u_{\bar{j} q} .
$$

From (3.13) and the definition of $\tilde{g}_{i \bar{j}}$,

$$
\begin{aligned}
\tilde{g}^{i \bar{i}} \tilde{g}_{1 \overline{1}, i \bar{i}}= & \tilde{g}^{i \bar{i}} \tilde{g}_{i \bar{i}, 1 \overline{1}}+\tilde{g}^{i \bar{i}}\left\{u_{1 \overline{1} i \bar{i}}-u_{i \bar{i} 1 \overline{1}}+a_{1, i \bar{i}} u_{\overline{1}}-a_{i, 1 \overline{1}} u_{\bar{i}}\right. \\
& +a_{\overline{1}, i \bar{i}} u_{1}-a_{\bar{i}, 1 \overline{1}} u_{i}+a_{1, i} u_{\overline{1 i}}-a_{i, 1} u_{\bar{i} \overline{1}}+a_{1, \bar{i}} u_{\overline{1} i}-a_{i, \overline{1}} u_{\bar{i} 1} \\
& +a_{\overline{1}, i} u_{1 \bar{i}}-a_{\bar{i}, 1} u_{i \overline{1}}+a_{\overline{1}, \bar{i}} u_{1 i}-a_{\bar{i}, \overline{1}} u_{i 1}+a_{1} u_{\overline{1} i \bar{i}}-a_{i} u_{\bar{i} 1 \overline{1}} \\
& \left.+a_{\overline{1}} u_{1 i \bar{i}}-a_{\bar{i}} u_{i 1 \overline{1}}\right\} \\
\geqslant & \tilde{g}^{i \bar{i}} \tilde{g}_{i \bar{i}, 1 \overline{1}}+\tilde{g}^{i \bar{i}}\left(\overline{T_{1 i}^{q}} u_{i \bar{q} 1}+T_{1 i}^{q} u_{\overline{1} q \bar{i}}+a_{1} u_{\overline{1} \bar{i} \bar{i}}-a_{i} u_{\bar{i} 1 \overline{1}}+a_{\overline{1}} u_{1 i \bar{i}}-a_{\bar{i}} u_{i 1 \overline{1}}\right) \\
& -\sum_{p} \tilde{g}^{i \bar{i}}\left(\left|u_{p \bar{i}}\right|^{2}+\left|u_{p i}\right|^{2}\right)-C\left(\operatorname{tr}_{\tilde{g}} g\right)\left(\operatorname{tr}_{g} \tilde{g}\right),
\end{aligned}
$$

where for the last line we used the assumption that $K \leqslant \lambda_{1} \leqslant \operatorname{tr}_{g} \tilde{g}$, and the uniform lower bound of $\operatorname{tr}_{g} \tilde{g}$ which follows from our equation (2.1).

Next, observe that

$$
u_{i \bar{j} k}=u_{k \bar{j} i}+T_{i k}^{p} u_{\overline{j p}}=u_{k i \bar{j}}+T_{i k}^{p} u_{\bar{j} p}-u_{p} R_{i \bar{j} k}{ }^{p} .
$$

Then, using this and (3.8),

$$
\begin{aligned}
\tilde{g}^{i \bar{i}}\left(a_{1} u_{\overline{1} i \bar{i}}+a_{\overline{1}} u_{1 i \bar{i}}\right)= & 2 \operatorname{Re}\left(\tilde{g}^{i \bar{i}} a_{\overline{1}} u_{1 i \bar{i}}\right)-a_{1} u_{\bar{q}} \tilde{g}^{i \bar{i}_{i}} R_{i \bar{i}}^{\bar{q} \overline{1}} \\
= & 2 \operatorname{Re}\left(\tilde{g}^{i \bar{i}} a_{\overline{1}}\left(u_{i \bar{i} 1}-T_{i 1}^{p} u_{\bar{i} p}+u_{p} R_{\overline{i \bar{i}}}^{p}\right)\right)-a_{1} u_{\bar{q}} \tilde{g}^{i \bar{i}} R_{\bar{i}} \overline{\bar{q}} \overline{1} \\
= & 2 \operatorname{Re}\left(a_{\overline{1}} F_{1}-\tilde{g}^{i \bar{i}} a_{\overline{1}}\left(T_{i 1}^{p} u_{\bar{i} p}-u_{p} R_{\bar{i} \overline{1} 1}^{p}\right.\right. \\
& \left.\left.+a_{i, 1} u_{\bar{i}}+a_{i} u_{1 \bar{i}}+a_{\bar{i}, 1} u_{i}+a_{\bar{i}} u_{i 1}\right)\right)-a_{1} u_{\bar{q}} \tilde{g}^{i \bar{i}} R_{\bar{i}} \bar{q} \overline{1}
\end{aligned}
$$


We also have

$$
\begin{aligned}
& \tilde{g}^{i \bar{i}}\left(\overline{T_{1 i}^{q}} u_{i \bar{q} 1}+T_{1 i}^{q} u_{\overline{1} q \bar{i}}\right) \\
= & 2 \operatorname{Re}\left(\tilde{g}^{i \bar{i}} \overline{T_{1 i}^{q}} u_{1 \bar{q} i}\right)+\tilde{g}^{i \bar{i}} \overline{T_{1 i}^{q}} T_{1 i}^{p} u_{\bar{q} p} \\
= & 2 \operatorname{Re}\left(\tilde{g}^{i \bar{i}} \overline{T_{1 i}^{q}}\left(\tilde{g}_{1 \bar{q}, i}-a_{1, i} u_{\bar{q}}-a_{1} u_{\bar{q} i}-a_{\bar{q}, i} u_{1}-a_{\bar{q}} u_{1 i}\right)\right) \\
& +\tilde{g}^{i \bar{i}} \overline{T_{1 i}^{q}} T_{i 1}^{p} u_{\bar{q} p} .
\end{aligned}
$$

Combining the above with (3.9) gives

$$
\begin{aligned}
\tilde{g}^{i \bar{i}} \tilde{g}_{1 \overline{1}, i \bar{i}} \geqslant & \tilde{g}^{i \bar{i}} \tilde{g}^{j \bar{j}} \tilde{g}_{i \bar{j}, 1} \tilde{g}_{j \bar{i}, \overline{1}}+2 \operatorname{Re}\left(\tilde{g}^{i \bar{i}} \overline{T_{1 i}^{q}} \tilde{g}_{1 \bar{q}, i}\right)-\tilde{g}^{i \bar{i}}\left\{a_{i} u_{\bar{i} 1 \overline{1}}+a_{\bar{i}} u_{i 1 \overline{1}}\right\} \\
& -2 \sum_{i, p} \tilde{g}^{i \bar{i}}\left(\left|u_{p \bar{i}}\right|^{2}+\left|u_{p i}\right|^{2}\right)-C\left(\operatorname{tr}_{\tilde{g}} g\right)\left(\operatorname{tr}_{g} \tilde{g}\right) .
\end{aligned}
$$

Next, using again Lemma 3.2 ,

$$
\begin{aligned}
2 \operatorname{Re}\left(\tilde{g}^{i \bar{i}} a_{\bar{i}} \frac{f_{i}}{\lambda_{1}}\right)= & 2 \operatorname{Re}\left(\tilde{g}^{i \bar{i}} a_{\bar{i}} \frac{\tilde{g}_{1 \overline{1}, i}}{\lambda_{1}}\right) \\
= & \frac{\tilde{g}^{i \bar{i}}}{\lambda_{1}}\left(a_{\bar{i}} u_{i 1 \overline{1}}+a_{i} u_{\bar{i} 1 \overline{1}}+a_{\bar{i}} T_{1 i}^{p} u_{\overline{1} p}-a_{\bar{i}} u_{p} R_{1 \overline{1} i}^{p}+a_{i} \overline{T_{1 i}^{q}} u_{1 \bar{q}}\right) \\
& +2 \operatorname{Re}\left(\frac{\tilde{g}^{i \bar{i}}}{\lambda_{1}} a_{\bar{i}}\left\{a_{1, i} u_{\overline{1}}+a_{1} u_{\overline{1} i}+a_{\overline{1}, i} u_{1}+a_{\overline{1}} u_{1 i}\right\}\right) \\
\geqslant & \frac{\tilde{g}^{i \bar{i}}}{\lambda_{1}}\left(a_{\bar{i}} u_{i 1 \overline{1}}+a_{i} u_{\bar{i} \overline{1} \overline{1}}\right)-\frac{1}{\lambda_{1}} \sum_{p} \tilde{g}^{i \bar{i}}\left(\left|u_{p \bar{i}}\right|^{2}+\left|u_{p i}\right|^{2}\right) \\
& -C \operatorname{tr}_{\tilde{g}} g
\end{aligned}
$$

and note that the terms involving three derivatives of $u$ exactly match those from (3.16), after multiplying by $-1 / \lambda_{1}$.

Now from (3.8) we have,

$$
\tilde{g}^{i \bar{i}} u_{i \bar{i} p} u_{\bar{p}}=F_{p} u_{\bar{p}}-\tilde{g}^{i \bar{i}} a_{i, p} u_{\bar{i}} u_{\bar{p}}-\tilde{g}^{i \bar{i}} a_{i} u_{\bar{i} p} u_{\bar{p}}-\tilde{g}^{i \bar{i}} a_{\bar{i}, p} u_{i} u_{\bar{p}}-\tilde{g}^{i \bar{i}} a_{\bar{i}} u_{i p} u_{\bar{p}} .
$$

Hence, making use of (3.15), and recalling that $\varphi^{\prime}=1 / K$,

$$
\begin{aligned}
& \varphi^{\prime} \tilde{g}^{i \bar{i}}\left(u_{p i \bar{i}} u_{\bar{p}}+u_{\bar{p} \bar{i}} u_{p}\right) \\
= & \varphi^{\prime} \tilde{g}^{i \bar{i}}\left(u_{i \bar{i} p} u_{\bar{p}}+u_{\bar{i} i \bar{p}} u_{p}+u_{r} u_{\bar{p}} R_{i \bar{i} p}^{r}-T_{i p}^{r} u_{\bar{p}} u_{\bar{i} r}+\overline{T_{p i}^{q}} u_{p} u_{i \bar{q}}\right) \\
= & 2 \varphi^{\prime} \operatorname{Re}\left(F_{p} u_{\bar{p}}-\tilde{g}^{i \bar{i}} a_{i, p} u_{\bar{i}} u_{\bar{p}}-\tilde{g}^{i \bar{i}} a_{i} u_{\bar{i} p} u_{\bar{p}}-\tilde{g}^{\bar{i}} a_{\bar{i}, p} u_{i} u_{\bar{p}}-\tilde{g}^{i \bar{i}} a_{\bar{i}} u_{i p} u_{\bar{p}}\right) \\
& +\varphi^{\prime} \tilde{g}^{i \bar{i}} u_{r} u_{\bar{p}} R_{i \bar{i} p}{ }^{r}-2 \varphi^{\prime} \operatorname{Re}\left(\tilde{g}^{i \bar{i}} T_{i p}^{r} u_{\bar{p}} u_{\bar{i} r}\right) \\
\geqslant & -\frac{\varphi^{\prime}}{4} \sum_{p} \tilde{g}^{i \bar{i}}\left(\left|u_{p \bar{i}}\right|^{2}+\left|u_{p i}\right|^{2}\right)-C \operatorname{tr}_{\tilde{g}} g .
\end{aligned}
$$


We also have

$$
2 \varphi^{\prime} \operatorname{Re}\left(\tilde{g}^{i \bar{i}} a_{\bar{i}}\left(u_{p} u_{\bar{p} i}+u_{p i} u_{\bar{p}}\right)\right) \geqslant-\frac{\varphi^{\prime}}{4} \sum_{p} \tilde{g}^{i \bar{i}}\left(\left|u_{p \bar{i}}\right|^{2}+\left|u_{p i}\right|^{2}\right)-C \operatorname{tr}_{\tilde{g}} g .
$$

Combining (3.11), (3.12), (3.16), (3.17), (3.18) and (3.19) gives

$$
\begin{aligned}
0 \geqslant & \frac{\tilde{g}^{i \bar{i}} \tilde{g}^{j \bar{j}} \tilde{g}_{i \bar{j}, 1} \tilde{g}_{j \bar{i}, \overline{1}}}{\lambda_{1}}+\sum_{q>\mu} \frac{\tilde{g}^{i \bar{i}}\left(\left|\tilde{g}_{1 \bar{q}, i}\right|^{2}+\left|\tilde{g}_{q \overline{1}, i}\right|^{2}\right)}{\lambda_{1}\left(\lambda_{1}-\lambda_{q}\right)}-\frac{\tilde{g}^{i \bar{i}}\left|\tilde{g}_{1 \overline{1}, i}\right|^{2}}{\lambda_{1}^{2}} \\
& +\frac{2 \operatorname{Re}\left(\tilde{g}^{i \bar{i}} \bar{T}_{1 i}^{q} \tilde{g}_{1 \bar{q}, i}\right)}{\lambda_{1}}+\left(\frac{1}{2} \varphi^{\prime}-\frac{C}{\lambda_{1}}\right) \sum_{p} \tilde{g}^{i \bar{i}}\left(\left|u_{p \bar{i}}\right|^{2}+\left|u_{p i}\right|^{2}\right) \\
& +\psi^{\prime \prime} \tilde{g}^{i \bar{i}}\left|u_{i}\right|^{2}+\psi^{\prime}\left(n-\operatorname{tr}_{\tilde{g}} g\right)-C \operatorname{tr}_{\tilde{g}} g
\end{aligned}
$$

for $C$ a universal constant (depending on $F$, $a$ etc).

We need to get a lower bound of

$$
\frac{\tilde{g}^{i \bar{i}} \tilde{g}^{j \bar{j}} \tilde{g}_{i \bar{j}, 1} \tilde{g}_{j \bar{i}, \overline{1}}}{\lambda_{1}}-\frac{\tilde{g}^{i \bar{i}}\left|\tilde{g}_{1 \overline{1}, i}\right|^{2}}{\lambda_{1}^{2}} \geqslant \sum_{i=2}^{n} \frac{\tilde{g}^{i \bar{i}} \tilde{g}_{i \overline{1}, 1} \tilde{g}_{1 \bar{i}, \overline{1}}}{\lambda_{1}^{2}}-\sum_{i=2}^{n} \frac{\tilde{g}^{i \bar{i}}\left|\tilde{g}_{1 \overline{1}, i}\right|^{2}}{\lambda_{1}^{2}},
$$

where we have discarded the terms with $j \neq 1$. But note that

$$
\tilde{g}_{i \overline{1}, 1}=\tilde{g}_{1 \overline{1}, i}+\lambda_{1} X_{1 \overline{1} i}
$$

where $X_{1 \overline{1} i}$ is defined by

$X_{1 \overline{1} i}:=\frac{1}{\lambda_{1}}\left(T_{i 1}^{p} u_{\overline{1} p}+a_{i, 1} u_{\overline{1}}+a_{i} u_{1 \overline{1}}+a_{\overline{1}, 1} u_{i}-a_{1, i} u_{\overline{1}}-a_{1} u_{i \overline{1}}-a_{\overline{1}, i} u_{1}+a_{\overline{1}} T_{1 i}^{k} u_{k}\right)$, and satisfies $\left|X_{1 \overline{1} i}\right| \leqslant C$ for a uniform $C$. In the above, we used (3.15) and the formula

$$
u_{i j}-u_{j i}=T_{j i}^{k} u_{k}
$$

Then

$$
\sum_{i=2}^{n} \frac{\tilde{g}^{i \bar{i}} \tilde{g}_{i \overline{1}, 1} \tilde{g}_{1 \bar{i}, \overline{1}}}{\lambda_{1}^{2}} \geqslant \sum_{i=2}^{n} \frac{\tilde{g}^{i \bar{i}}\left|\tilde{g}_{1 \overline{1}, i}\right|^{2}}{\lambda_{1}^{2}}+2 \operatorname{Re}\left(\sum_{i=2}^{n} \frac{\tilde{g}^{i \bar{i}} g_{1 \overline{1}, i} \overline{X_{1 \overline{1} i}}}{\lambda_{1}}\right) .
$$

To deal with the second term, we use (3.10) to compute

$$
\begin{aligned}
& 2 \operatorname{Re}\left(\sum_{i=2}^{n} \frac{\tilde{g}^{i \bar{i}} \tilde{g}_{1 \overline{1}, i} \overline{X_{1 \overline{1} i}}}{\lambda_{1}}\right) \\
= & -2 \operatorname{Re}\left(\sum_{i=2}^{n} \tilde{g}^{i \bar{i}}\left(\varphi^{\prime}\left(u_{p} u_{\bar{p} i}+u_{p i} u_{\bar{p}}\right)+\psi^{\prime} u_{i}\right) \overline{X_{1 \overline{1} i}}\right) \\
\geqslant & -\frac{\varphi^{\prime}}{8} \sum_{p} \tilde{g}^{i \bar{i}}\left(\left|u_{p \bar{i}}\right|^{2}+\left|u_{p i}\right|^{2}\right)-C \operatorname{tr}_{\tilde{g}} g+\psi^{\prime}\left(C \tilde{g}^{i \bar{i}}\left|u_{i}\right|^{2}+\frac{1}{4} \operatorname{tr}_{\tilde{g}} g\right),
\end{aligned}
$$

where we recall that $\psi^{\prime}<0$. 
Next we deal with the fourth term on the right hand side of (3.20). From Lemma 3.2 we have $\tilde{g}_{1 \bar{q}, i}=0$ for $1<q \leqslant \mu$ and hence

$$
\frac{2 \operatorname{Re}\left(\tilde{g}^{i \bar{i}} \bar{T}_{1 i}^{q} \tilde{g}_{1 \bar{q}, i}\right)}{\lambda_{1}}=\frac{2 \operatorname{Re}\left(\tilde{g}^{i \bar{i}} \bar{T}_{1 i}^{1} \tilde{g}_{1 \overline{1}, i}\right)}{\lambda_{1}}+2 \sum_{q>\mu} \frac{\operatorname{Re}\left(\tilde{g}^{i \bar{i}} \bar{T}_{1 i}^{q} \tilde{g}_{1 \bar{q}, i}\right)}{\lambda_{1}}
$$

But using the same argument as in (3.23), replacing $\left|X_{1 \bar{i} i}\right| \leqslant C$ by $\left|T_{1 i}^{1}\right| \leqslant C$, we obtain

$$
\begin{aligned}
\frac{2 \operatorname{Re}\left(\tilde{g}^{i \bar{i} T_{1 i}^{1}} \tilde{g}_{1 \overline{1}, i}\right)}{\lambda_{1}} \geqslant & -\frac{\varphi^{\prime}}{8} \sum_{p} \tilde{g}^{i \bar{i}}\left(\left|u_{p \bar{i}}\right|^{2}+\left|u_{p i}\right|^{2}\right)-C \operatorname{tr}_{\tilde{g}} g \\
& +\psi^{\prime}\left(C \tilde{g}^{i \bar{i}}\left|u_{i}\right|^{2}+\frac{1}{4} \operatorname{tr}_{\tilde{g}} g\right) .
\end{aligned}
$$

On the other hand we have

$$
\begin{aligned}
2 \sum_{q>\mu} \frac{\operatorname{Re}\left(\tilde{g}^{i \bar{i}} \bar{T}_{1 i}^{q} \tilde{g}_{1 \bar{q}, i}\right)}{\lambda_{1}} & \geqslant-\sum_{q>\mu} \frac{\tilde{g}^{i \bar{i}}\left|\tilde{g}_{1 \bar{q}, i}\right|^{2}}{\lambda_{1}\left(\lambda_{1}-\lambda_{q}\right)}-\sum_{q>\mu} \tilde{g}^{i \bar{i}}\left|T_{1 i}^{q}\right|^{2} \frac{\left(\lambda_{1}-\lambda_{q}\right)}{\lambda_{1}} \\
& \geqslant-\sum_{q>\mu} \frac{\tilde{g}^{i \bar{i}}\left|\tilde{g}_{1 \bar{q}, i}\right|^{2}}{\lambda_{1}\left(\lambda_{1}-\lambda_{q}\right)}-C \operatorname{tr}_{\tilde{g}} g .
\end{aligned}
$$

Combining (3.20) with (3.21), (3.22), (3.23), (3.24), (3.25) and (3.26) we obtain for a uniform constant $C$,

$$
\begin{aligned}
0 \geqslant & \left(\frac{1}{4} \varphi^{\prime}-\frac{C}{\lambda_{1}}\right) \sum_{i, p} \tilde{g}^{i \bar{i}}\left(\left|u_{p \bar{i}}\right|^{2}+\left|u_{p i}\right|^{2}\right)+\left(-\psi^{\prime} / 2-C\right) \operatorname{tr}_{\tilde{g}} g \\
& +\left(\psi^{\prime \prime}+C \psi^{\prime}\right) \tilde{g}^{i \bar{i}}\left|u_{i}\right|^{2}+\psi^{\prime} n .
\end{aligned}
$$

But since we may assume that $\lambda_{1} \geqslant 4 C K$, the first term on the right hand side is nonnegative. Pick $A=2(C+1)$ so that $-\psi^{\prime} / 2-C \geqslant 1$ and $\psi^{\prime \prime}+C \psi^{\prime} \geqslant$ 0 . Then $\operatorname{tr}_{\tilde{g}} g$ and hence $\lambda_{1}$ is uniformly bounded from above at the maximum of $Q$, and the result follows.

Remark. In the proof above we used a viscosity type argument to deal with the non-differentiability of the largest eigenvalue $\lambda_{1}$. There are other methods to deal with this issue: one is to use a perturbation argument as in [26, 27]; another is to replace $\lambda_{1}$ by a carefully chosen quadratic function of $\tilde{g}_{i \bar{j}}$ as in 33 .

\section{Proof of the Main theorem}

4.1. Higher order estimates. First, we discuss the a priori higher order estimates, in the same setting as Theorems 2.1 and 3.1. Thanks to the estimates in these Theorems, a blowup argument can be employed exactly as in [8, 26, 27, 31] to obtain that $\sup _{M}|\partial u|_{g} \leqslant C$, and therefore also $\sup _{M} \operatorname{tr}_{g} \tilde{g} \leqslant C$. Here we use the classical Liouville Theorem stating that a 
bounded plurisubharmonic function on $\mathbb{C}^{n}$ is constant (indeed, by restricting to complex lines, this reduces to the well-known fact that a bounded subharmonic function in $\mathbb{C}$ is constant).

The PDE (2.1) then implies that $\tilde{g}$ is uniformly equivalent to $g$, at which point we can then apply the Evans-Krylov theory [9, 20, 34] (see also [28]) to obtain uniform a priori $C^{2, \alpha}$ bounds on $u$, for some uniform $0<\alpha<1$. Differentiating the equation and using Schauder theory, we then deduce uniform a priori $C^{k}$ bounds for all $k \geqslant 0$.

4.2. Existence of a solution. We employ the continuity method. For $t \in[0,1]$ we consider the family of equations for $\left(u_{t}, b_{t}\right)$

$$
\begin{aligned}
& \operatorname{det}\left(g_{i \bar{j}}+a_{i} u_{t, \bar{j}}+a_{\bar{j}} u_{t, i}+u_{t, i \bar{j}}\right)=e^{t F+b_{t}} \operatorname{det}\left(g_{i \bar{j}}\right), \\
& \text { with }\left(g_{i \bar{j}}+a_{i} u_{t, \bar{j}}+a_{\bar{j}} u_{t, i}+u_{t, i \bar{j}}\right)>0 .
\end{aligned}
$$

Suppose we have a solution for $t=\hat{t}$ and write

$$
\hat{\omega}=\omega+\sqrt{-1} a \wedge \bar{\partial} u_{\hat{t}}-\sqrt{-1} \bar{a} \wedge \partial u_{\hat{t}}+\sqrt{-1} \partial \bar{\partial} u_{\hat{t}},
$$

and $\hat{H}$ for the linearized operator defined as in (2.5). By the same argument of Gauduchon [14] that was mentioned earlier, we may find a smooth function $v$, normalized by $\int_{M} e^{v} \hat{\omega}^{n}=1$, such that

$$
\int_{M} \hat{H}(\psi) e^{v} \hat{\omega}^{n}=0
$$

for all smooth functions $\psi$, i.e. $e^{v}$ generates the kernel of the adjoint $\hat{H}^{*}$ of $\hat{H}$ (with respect to the $L^{2}$ inner product with volume form $\hat{\omega}^{n}$ ). Fix $0<\alpha<1$ and consider the operator

$$
\begin{aligned}
\Upsilon(\psi) & =\log \frac{(\hat{\omega}+\sqrt{-1} a \wedge \bar{\partial} \psi-\sqrt{-1} \bar{a} \wedge \partial \psi+\sqrt{-1} \partial \bar{\partial} \psi)^{n}}{\hat{\omega}^{n}} \\
& -\log \left(\int_{M} e^{v}(\hat{\omega}+\sqrt{-1} a \wedge \bar{\partial} \psi-\sqrt{-1} \bar{a} \wedge \partial \psi+\sqrt{-1} \partial \bar{\partial} \psi)^{n}\right),
\end{aligned}
$$

mapping $C^{3, \alpha}$ functions $\psi$ with zero average (and such that $\hat{\omega}+\sqrt{-1} a \wedge$ $\bar{\partial} \psi-\sqrt{-1} \bar{a} \wedge \partial \psi+\sqrt{-1} \partial \bar{\partial} \psi>0)$ to the space of $C^{1, \alpha}$ functions $w$ satisfying $\int_{M} e^{w+v} \hat{\omega}^{n}=1$ (whose tangent space at 0 consists precisely of $C^{1, \alpha}$ functions orthogonal to the kernel of $\hat{H}^{*}$ ). For any $C^{3, \alpha}$ function $\zeta$ we have

$$
\int_{M} e^{v} \hat{H}(\zeta) \hat{\omega}^{n}=\int_{M} \zeta \hat{H}^{*}\left(e^{v}\right) \hat{\omega}^{n}=0
$$

hence the linearization of $\Upsilon$ at 0 is $\hat{H}$. Thanks to the Fredholm alternative, $\hat{H}$ is an isomorphism of the tangent spaces, and so the Inverse Function Theorem provides us with $C^{3, \alpha}$ functions $\psi_{t}$ for $t$ near $\hat{t}$ which satisfy

$$
\Upsilon\left(\psi_{t}\right)=(t-\hat{t}) F-\log \left(\int_{M} e^{(t-\hat{t}) F} e^{v} \hat{\omega}^{n}\right),
$$


so that $u_{t}=u_{\hat{t}}+\psi_{t}$ solve (4.1) for some $b_{t} \in \mathbb{R}$. Lastly, differentiating (4.1) and using Schauder estimates and bootstrapping, we easily see that our $C^{3, \alpha}$ solutions are in fact smooth.

This establishes that the set of all $t \in[0,1]$ for which we have a solution $\left(u_{t}, b_{t}\right)$ of (4.1) is open (and nonempty, since we can take $\left.\left(u_{0}, b_{0}\right)=(0,0)\right)$. At this point we can also impose that $\sup _{M} u_{t}=0$ by adding a $t$-dependent constant. To show that the set of such $t \in[0,1]$ is also closed, it suffices to prove a priori estimates for $u_{t}$ (in $C^{k}$ for all $k \geqslant 0$ ) and $b_{t}$. The bound $\left|b_{t}\right| \leqslant$ $\sup _{M}|F|$ is elementary by the maximum principle, and then the estimates for $u_{t}$ follow from section 4.1 above.

4.3. Uniqueness. In the setting of the main theorem 1.1. uniqueness of $b$ and $u$ follows from a simple maximum principle argument, see e.g. [7.

\section{REFERENCES}

[1] Bedford, E. Survey of pluri-potential theory, in Several complex variables (Stockholm, 1987/1988), 48-97, Math. Notes 38, Princeton Univ. Press, Princeton, NJ, 1993.

[2] Błocki, Z., On uniform estimate in Calabi-Yau theorem, Sci. China Ser. A 48 (2005), suppl., 244-247.

[3] Błocki, Z., On the uniform estimate in the Calabi-Yau theorem, II, Sci. China Math. 54 (2011), no.7, 1375-1377.

[4] Błocki, Z., A gradient estimate in the Calabi-Yau theorem, Math. Ann. 344 (2009), no. 2, 317-327.

[5] Brendle, S., Choi, K. and Daskalopoulos, P., Asymptotic behavior of flows by powers of the Gaussian curvature, Acta Math. 219 (2017), no.1, 1-16.

[6] Cherrier, P., Équations de Monge-Ampère sur les variétés Hermitiennes compactes, Bull. Sc. Math (2) 111 (1987), 343-385.

[7] Chu, J., Tosatti, V. and Weinkove, B., The Monge-Ampère equation for non-integrable almost complex structures, to appear in J. Eur. Math. Soc. (JEMS).

[8] Dinew, S. and Kołodziej, S., Liouville and Calabi-Yau type theorems for complex Hessian equations, Amer. J. Math. 139 (2017), no.2, 403-415.

[9] Evans, L.C., Classical solutions of fully nonlinear, convex, second order elliptic equations, Comm. Pure Appl. Math. 25 (1982), 333-363.

[10] Feng, K., Ge, H. and Zheng, T., The Dirichlet problem of fully nonlinear equations on Hermitian manifolds, preprint, arXiv:1905.02412

[11] Fu, J., Wang, Z. and Wu, D., Form-type Calabi-Yau equations, Math. Res. Lett. 17 (2010), no.5, 887-903.

[12] Fu, J., Wang, Z. and Wu, D., Form-type Calabi-Yau equations on Kähler manifolds of nonnegative orthogonal bisectional curvature, Calc. Var. Partial Differential Equations 52 (2015), no.1-2, 327-344.

[13] Fu, J. and Yau, S.-T., The theory of superstring with flux on non-Kähler manifolds and the complex Monge-Ampère equation, J. Differential Geom. 78 (2008), no.3, 369428.

[14] Gauduchon, P., Le théorème de l'excentricité nulle, C. R. Acad. Sci. Paris Sér. A-B 285 (1977), no.5, A387-390.

[15] Gauduchon, P., La 1-forme de torsion d'une variété hermitienne compacte, Math. Ann. 267 (1984), 495-518.

[16] Guan, B. and Li, Q., Complex Monge-Ampère equations and totally real submanifolds, Adv. Math. 225 (2010), no.3, 1185-1223. 
[17] Guan, B. and Nie, X., Fully nonlinear elliptic equations with gradient terms on Hermitian manifolds, preprint.

[18] Harvey, F. R. and Lawson, H. B., Geometric plurisubharmonicity and convexity: an introduction, Adv. Math. 230 (2012), no.4-6, 2428-2456.

[19] Hou, Z., Ma, X.-N. and Wu, D., A second order estimate for complex Hessian equations on a compact Kähler manifold, Math. Res. Lett. 17 (2010), no.3, 547-561.

[20] Krylov, N.V., Boundedly nonhomogeneous elliptic and parabolic equations, Izvestia Akad. Nauk. SSSR 46 (1982), 487-523; English translation in. Math. USSR Izv. 20 (1983), no. 3, 459-492.

[21] Phong, D.H., Picard, S. and Zhang, X., A second order estimate for general complex Hessian equations, Anal. PDE 9 (2016), no.7, 1693-1709.

[22] Phong, D.H., Picard, S. and Zhang, X., The Fu-Yau equation with negative slope parameter, Invent. Math. 209 (2017), no.2, 541-576.

[23] Phong, D.H., Picard, S. and Zhang, X., New curvature flows in complex geometry, in Surveys in differential geometry 2017. Celebrating the 50th anniversary of the Journal of Differential Geometry, 331-364, Surv. Differ. Geom., 22, Int. Press, Somerville, MA, 2018.

[24] Phong, D.H., Picard, S. and Zhang, X., Fu-Yau Hessian equations, preprint, arXiv:1801.09842.

[25] Popovici, D., Aeppli cohomology classes associated with Gauduchon metrics on compact complex manifolds, Bull. Soc. Math. France 143 (2015), no.4, 763-800.

[26] Székelyhidi, G., Fully non-linear elliptic equations on compact Hermitian manifolds, J. Differential Geom. 109 (2018), no.2, 337-378

[27] Székelyhidi, G., Tosatti, V. and Weinkove, B., Gauduchon metrics with prescribed volume form, Acta Math. 219 (2017), no.1, 181-211.

[28] Tosatti, V., Wang, Y., Weinkove, B. and Yang, X., $C^{2, \alpha}$ estimates for nonlinear elliptic equations in complex and almost complex geometry, Calc. Var. Partial Differential Equations 54 (2015), no.1, 431-453.

[29] Tosatti, V. and Weinkove, B., Estimates for the complex Monge-Ampère equation on Hermitian and balanced manifolds, Asian J. Math. 14 (2010), no.1, 19-40.

[30] Tosatti, V. and Weinkove, B., The complex Monge-Ampère equation on compact Hermitian manifolds, J. Amer. Math. Soc. 23 (2010), no.4, 1187-1195.

[31] Tosatti, V. and Weinkove, B., The Monge-Ampère equation for $(n-1)$ plurisubharmonic functions on a compact Kähler manifold, J. Amer. Math. Soc. 30 (2017), no.2, 311-346.

[32] Tosatti, V. and Weinkove, B., The Aleksandrov-Bakelman-Pucci estimate and the Calabi-Yau equation, in Nonlinear Analysis in Geometry and Applied Mathematics, Part 2, 147-158, Harvard CMSA Ser. Math. 2, International Press, 2018.

[33] Tosatti, V. and Weinkove, B., Hermitian metrics, $(n-1, n-1)$ forms and MongeAmpère equations, to appear in J. Reine Angew. Math.

[34] Trudinger, N.S., Fully nonlinear, uniformly elliptic equations under natural structure conditions, Trans. Am. Math. Soc. 278 (1983), no. 2, 751-769.

[35] Yau, S.-T., On the Ricci curvature of a compact Kähler manifold and the complex Monge-Ampère equation, I, Comm. Pure Appl. Math. 31 (1978), no.3, 339-411.

[36] Yuan, R., On a class of fully nonlinear elliptic equations containing gradient terms on compact Hermitian manifolds, Canad. J. Math. 70 (2018), no.4, 943-960.

[37] Zhang, X. A priori estimates for complex Monge-Ampère equation on Hermitian manifolds, Int. Math. Res. Not. IMRN 2010, 19, 3814-3836.

[38] Zheng, T., A parabolic Monge-Ampère type equation of Gauduchon metrics, to appear in Int. Math. Res. Not. IMRN.

Department of Mathematics, Northwestern University, 2033 Sheridan Road, EvANSTON, IL 60208 